\title{
Retinal Vessel Tree as Biometric Pattern
}

\author{
Marcos Ortega and Manuel G. Penedo \\ University of Coruña \\ Department of Computer Science \\ Spain
}

\section{Introduction}

In current society, reliable authentication and authorization of individuals are becoming more and more necessary tasks for everyday activities or applications. Just for instance, common situations such as accessing to a building restricted to authorized people (members, workers,...), taking a flight or performing a money transfer require the verification of the identity of the individual trying to perform these tasks. When considering automation of the identity verification, the most challenging aspect is the need of high accuracy, in terms of avoiding incorrect authorizations or rejections. While the user should not be denied to perform a task if authorized, he/she should be also ideally inconvenienced to a minimum which further complicates the whole verification process Siguenza Pizarro \& Tapiador Mateos (2005).

With this scope in mind, the term biometrics refers to identifying an individual based on his/her distinguished intrinsic characteristics. Particularly, this characteristics usually consist of physiological or behavioral features. Physiological features, such as fingerprints, are physical characteristics usually measured at a particular point of time. Behavioral characteristics, such as speech or handwriting, make reference to the way some action is performed by every individual. As they characterize a particular activity, behavioral biometrics are usually measured over time and are more dependant on the individual's state of mind or deliberated alteration. To reinforce the active versus passive idea of both paradigms, physiological biometrics are also usually referred to as static biometrics while behavioral ones are referred to as dynamic biometrics.

The traditional authentication systems based on possessions or knowledge are widely spread in the society but they have many drawbacks that biometrics try to overcome. For instance, in the scope of the knowledge-based authentication, it is well known that password systems are vulnerable mainly due to the wrong use of users and administrators. It is not rare to find some administrators sharing the same password, or users giving away their own to other people. One of the most common problems is the use of easily discovered passwords (child names, birth dates, car plate,...). On the other hand, the use of sophisticated passwords consisting of numbers, upper and lower case letters and even punctuation marks makes it harder to remember them for an user.

Nevertheless, the password systems are easily broken by the use of brute force where powerful computers generate all the possible combinations and test it against the authentication system. In the scope of the possession-based authentication, it is obvious that the main concerns are 
related to the loss of the identification token. If the token was stolen or found by another individual, the privacy and/or security would be compromised. Biometrics overcome most of these concerns while they also allow an easy entry to computer systems to non expert users with no need to recall complex passwords. Additionally, commercial webs on the Internet are favored not only by the increasing trust being transmitted to the user but also by the possibility of offering a customizable environment for every individual along with the valuable information on personal preferences for each of them.

Many different human biometrics have been used to build a valid template for verification and identification tasks. Among the most common biometrics, we can find the fingerprint Bolle et al. (2002); Maio \& Maltoni (1997); Seung-Hyun et al. (1995); Venkataramani \& Kumar (2003), iris Chou et al. (2006); He et al. (2008); Kim, Cho, Choi \& Marks (2004); Ma et al. (2002); Nabti \& Bouridane (2007) or face Kim, Kim, Bang \& Lee (2004); Kisku et al. (2008); Mian et al. (2008); Moghaddam \& Pentland (1997); Yang et al. (2000) or hand geometry Jain et al. (1999); Sidlauskas (1988); systems Lab (n.d.); Zunkel (1999). However, there exist other emerging biometrics where we can find retina biometrics. Identity verification based on retina uses the blood vessels pattern present in the retina (Figure1).

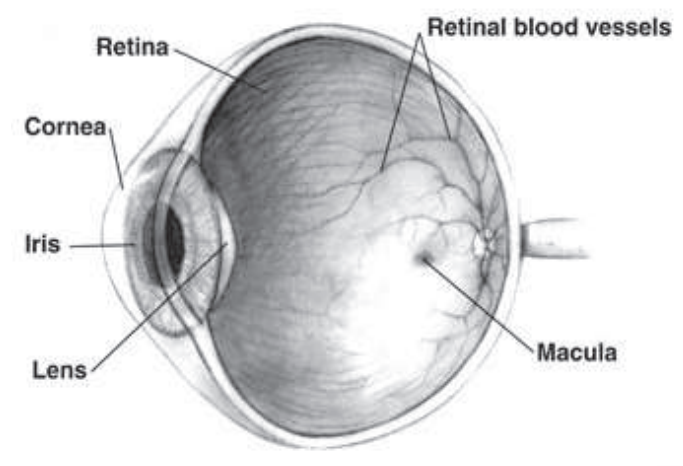

Fig. 1. Schema of the retina in the human eye. Blood vessels are used as biometric characteristic.

Retinal blood vessel pattern is unique for each human being even in the case of identical twins. Moreover, it is a highly stable pattern over time and totally independent of genetic factors. Also, it is one of the hardest biometric to forge as the identification relies on the blood circulation along the vessels. These property make it one of the best biometric characteristic in high security environments. Its main drawback is the acquisition process which requires collaboration from the user and it is sometimes perceived as intrusive. As it will be further discussed, some advances have been done in this field but, in any case, this continues to be the weak point in retinal based authentication.

Robert Hill introduced the first identification system based on retina Hill (1999). The general idea was that of taking advantage of the inherent properties of the retinal vessel pattern to build a secure system. The system acquired the data via a scanner that required the user to be still for a few seconds. The scanner captured a band in the blood vessels area similar to the one employed in the iris recognition as shown in Figure 2.

The scanned area is a circular band around blood vessels. This contrast information of this area is processed via fast Fourier transform. The transformed data forms the final biometric pattern 


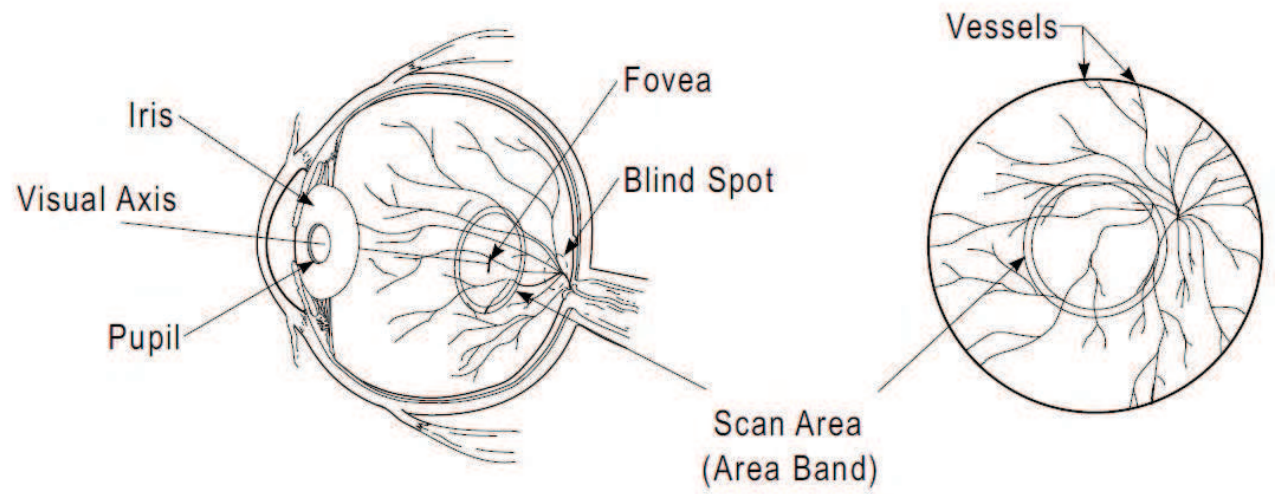

Fig. 2. Illustration of the scan area in the retina used in the system of Robert Hill.

considered in this system. This pattern worked good enough as the acquisition environment was very controlled. Of course, this is also the source of the major drawbacks present in the device: the data acquisition process. This process was both slow and uncomfortable for the user. Moreover, the hardware was very expensive and, therefore, it rendered the system hardly appealing. Finally, the result was that the use of retinal pattern as a biometric characteristic, despite all its convenient properties, was discontinued.

Nowadays, retinal image cameras (Figure 3) are capable of taking a photograph of the retina area in a human eye without any intrusive or dangerous scanning. Also, currently, the devices are cheaper and more accessible in general. This technology reduces the perception of danger by the user during the retina acquisition process but also brings more freedom producing a more heterogeneous type of retinal images to work with. The lighting conditions and the movement of the user's eye vary between acquisitions. This produces as a result that previous systems based on contrast information of reduced areas may lack the required precision in some cases, increasing the false rejection rate.
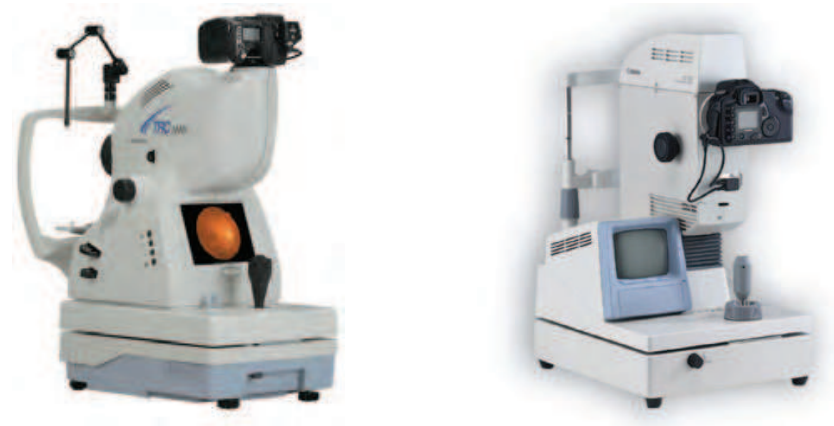

Fig. 3. Two retinal image cameras. The retinal image is acquired by taking an instant photograph.

In Figure 4 it can be observed two images from the same person acquired at different times by the same retinograph. There are some zones in the retinal vessels that can not be compared 
because of the lack of information in one of the images. Thus, to allow the retinal biometrics to keep and increase the acquisition comfortability, it is necessary to implement a more robust methodology that, maintaining the extremely low error rates, is capable to cope with a more heterogeneous range of retinal images.
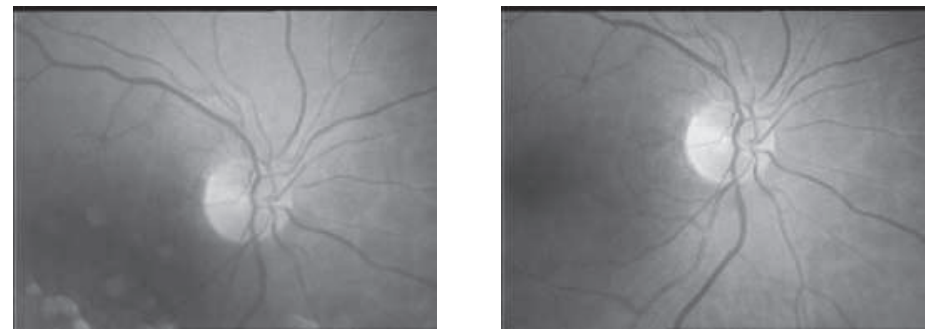

Fig. 4. Example of two digital retina images from the same individual acquired by the same retinal camera at different times.

This work is focused on the proposal of a novel personal authentication system based on the retinal vessel tree. This system deals with the new challenges in the retinal field where a more robust pattern has to be designed in order to increase the usability for the acquisition stage. In this sense, the approach presented here to the retinal recognition is closer to the fingerprint developments than to the iris ones as the own structure of the retinal vessel tree suggests. Briefly, the objectives of this work are enumerated:

- Empirical evaluation of the retinal vessel tree as biometric pattern

- Design a robust, easy to store and process biometric pattern making use of the whole retinal vessel tree information

- Development of an efficient and effective methodology to compare and match such retinal patterns

- Analysis on similarity metrics performance to establish reliable thresholds in the authentication process

To deal with the suggested goals, the rest of this document is organized as follows. Second section introduces previous works and research on the retinal vessel tree as biometric pattern. Section 3 presents the methodology developed to build the authentication system, including biometric template construction and template matching algorithms. Section 4 discusses the experiments aimed to test the proposed methodologies, including an analysis of similarity measures. Finally, Section 5 offers some conclusions and final discussion.

\section{Related work}

Awareness of the uniqueness of the retinal vascular pattern dates back to 1935 when two ophthalmologists, Drs. Carleton Simon and Isodore Goldstein, while studying eye disease, realized that every eye has its own unique pattern of blood vessels. They subsequently published a paper on the use of retinal photographs for identifying people based on their blood vessel patterns Simon \& Goldstein (1935). Later in the 1950s, their conclusions were supported by Dr. Paul Tower in the course of his study of identical twins. He noted that, of any two persons, identical twins would be the most likely to have similar retinal vascular 
patterns. However, Tower showed that, of all the factors compared between twins, retinal vascular patterns showed the least similarities Tower (1955).

Blood vessels are among the first organs to develop and are entirely derived from the mesoderm. Vascular development occurs via two processes termed vasculogenesis and angiogenesis. Vasculogenesis, this is, the blood vessel assembly during embryogenesis, begins with the clustering of primitive vascular cells or hemangioblasts into tube-like endothelial structures, which define the pattern of the vasculature. In angiogenesis, new vessels arise by sprouting of budlike and fine endothelial extensions from preexisting vessels Noden (1989). In a more recent study Whittier et al. (2003), retinal vascular pattern images from livestock were digitally acquired in order to evaluate their pattern uniqueness. To evaluate each retinal vessel pattern, the dominate trunk vessel of bovine retinal images was positioned vertically and branches on the right and left of the trunk and other branching points were evaluated. Branches from the left (mean 6.4 and variance 2.2) and the right (mean 6.4 and variance 1.5) of the vascular trunk; total branches from the vascular trunk (mean 12.8 and variance 4.3), and total branching points (mean 20.0 and variance 13.2) showed differences across all animals (52). A paired comparison of the retinal vessel patterns from both eyes of 30 other animals confirmed that eyes from the same animal differ. Retinal images of 4 cloned sheep from the same parent line were evaluated to confirm the uniqueness of the retinal vessel patterns in genetically identical animals. This would be confirming the uniqueness of animal retinal vascular pattern suggested earlier in the 1980s also by De Schaepdrijver et al. (1989).

In general, retinal vessel tree his is a unique pattern in each individual and it is almost impossible to forge that pattern in a false individual. Of course, the pattern does not change through the individual's life, unless a serious pathology appears in the eye. Most common diseases like diabetes do not change the pattern in a way that its topology is affected. Some lesions (points or small regions) can appear but they are easily avoided in the vessels extraction method that will be discussed later. Thus, retinal vessel tree pattern has been proved a valid biometric trait for personal authentication as it is unique, time invariant and very hard to forge, as showed by Mariño et al. C.Mariño et al. (2003); Mariño et al. (2006), who introduced a novel authentication system based on this trait. In that work, the whole arterial-venous tree structure was used as the feature pattern for individuals. The results showed a high confidence band in the authentication process but the database included only 6 individuals with 2 images for each of them. One of the weak points of the proposed system was the necessity of storing and handling a whole image as the biometric pattern. This greatly difficults the storing of the pattern in databases and even in different devices with memory restrictions like cards or mobile devices. In Farzin et al. (2008) a pattern is defined using the optic disc as reference structure and using multi scale analysis to compute a feature vector around it. Good results were obtained using an artificial scenario created by randomly rotating one image per user for different users. The dataset size is 60 images, rotated 5 times each. The performance of the system is about a $99 \%$ accuracy. However, the experimental results do not offer error measures in a real case scenario where different images from the same individual are compared.

Based on the idea of fingerprint minutiae, a robust pattern is introduced where a set of landmarks (bifurcations and crossovers of retinal vessel tree) were extracted and used as feature points. In this scenario, the pattern matching problem is reduced to a point pattern matching problem and the similarity metric has to be defined in terms of matched points. A common problem in previous approaches is that the optic disc is used as a reference structure in the image. The detection of the optic disc is a complex problem and in some individuals 
with eye diseases this cannot be achieved correctly. In this work, the use of reference structures is avoided to allow the system to cope with a wider range of images and users.

\section{Retinal verification based on feature points}

Figure 5 illustrates the general schema for the new feature point based authentication approach. The newly introduced stages are the feature point extraction and the feature point matching. The following chapter sections will discuss the methodology on these new stages of the system.

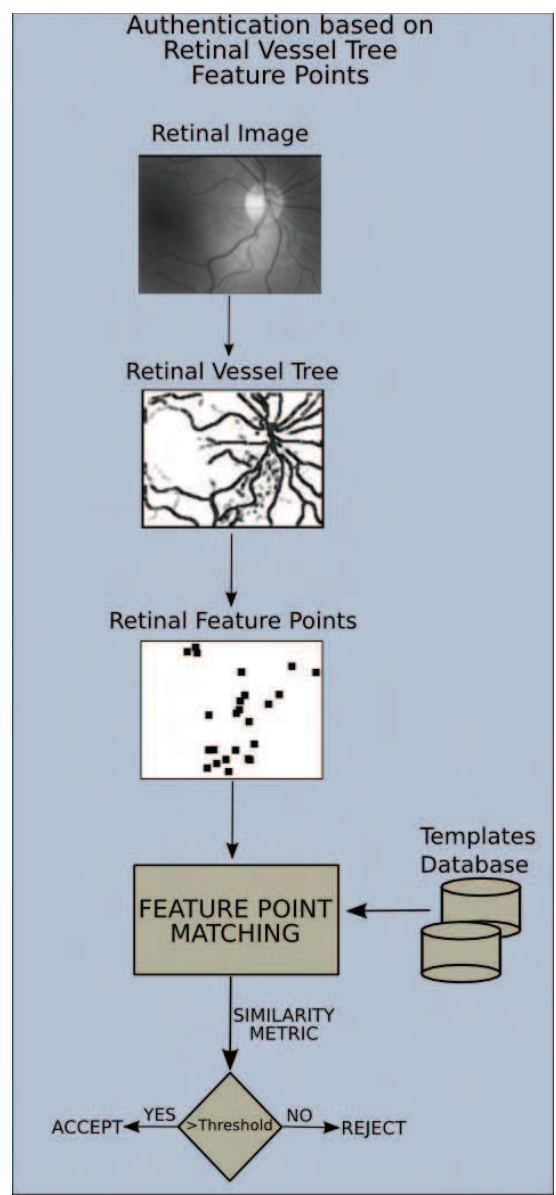

Fig. 5. Schema of the main stages for the authentication system based in the retinal vessel tree structure.

\subsection{Feature points extraction}

Following the idea that vessels can be thought of as creases (ridges or valleys) when images are seen as landscapes (see Figure 6), curvature level curves will be used to calculate the creases 
(ridge and valley lines). Several methods for crease detection have been proposed in the literature (see López et al. (1999) for a comparison between methods), but finally a differential geometry based method López et al. (2000) was selected because of its good performance in similar images Lloret et al. $(1999 ; 2001)$, producing very good results.

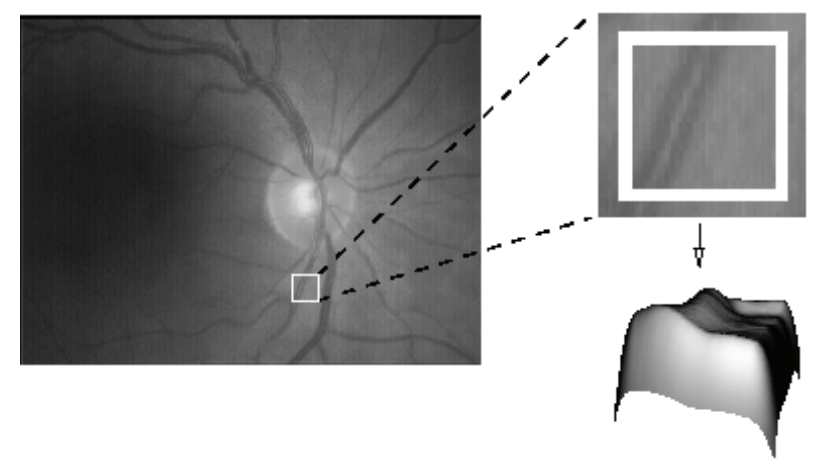

Fig. 6. Picture of a region of the retinal image as landscape. Vessels can be represented as creases.

Among the many definitions of crease, the one based on Level Set Extrinsic Curvature, LSEC López et al. (1998), has useful invariance properties. The geometry based method named LSEC gives rise to several problems, solved through the improvement of this method by a multilocal solution, the MLSECLópez et al. (2000). But results obtained with MLSEC can still be improved by pre-filtering the image gradient vector field using structure tensor analysis and by discarding creaseness at isotropic areas by means of the computation of a confidence measure. The methodology allows to tune several parameters to apply such filters as for creases with a concrete width range or crease length. In Caderno et al. (2004) a methodology was presented for automatic parameter tuning by analyzing contrast variance in the retinal image.

One of the main advantages of this method is that it is invariant to changes in contrast and illumination, allowing the extraction of creases from arteries and veins independently of the characteristics of the images, avoiding a previous normalization of the input images. The final result is an image where the retinal vessel tree is represented by its crease lines. Figure 7 shows several examples of the creases obtained from different retinal images.

The landmarks of interest are points where two different vessels are connected. Therefore, it is necessary to study the existing relationships between vessels in the image. The first step is to track and label the vessels to be able to establish their relationships between them.

In Figure 8, it can be observed that the crease images show discontinuities in the crossovers and bifurcations points. This occurs because of the two different vessels (valleys or ridges) coming together into a region where the crease direction can not be set. Moreover, due to some illumination or intensity loss issues, the crease images can also show some discontinuities along a vessel (Figure 8). This issue requires a process of joining segments to build the whole vessels prior to the bifurcation/crossover analysis.

Once the relationships between segments are established, a final stage will take place to remove some possible spurious feature points. Thus, the four main stages in the feature point extraction process are: 

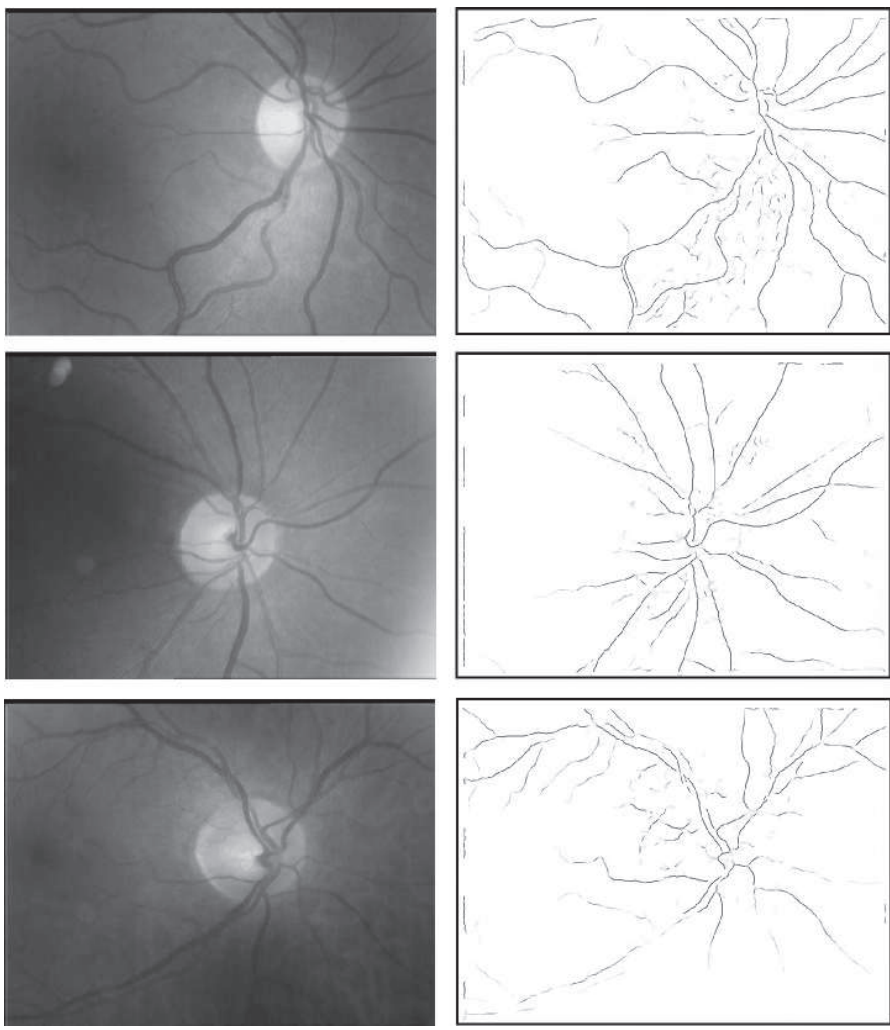

Fig. 7. Three examples of digital retinal images, showing the variability of the vessel tree among individuals. Left column: input images. Right column: creases of images on the left column representing the main vessels.

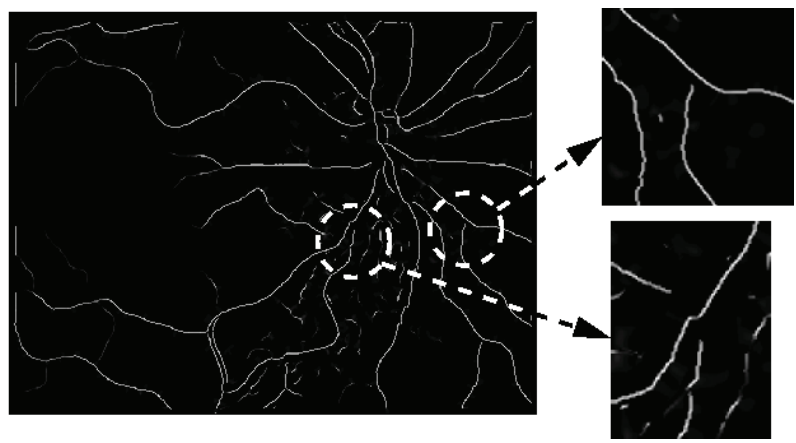

Fig. 8. Example of discontinuities in the creases of the retinal vessels. Discontinuities in bifurcations and crossovers are due to two creases with different directions joining in the same region. But, also, some other discontinuities along a vessel can happen due to illumination and contrast variations in the image. 
1. Labelling of the vessels segments

2. Establishing the joint or union relationships between vessels

3. Establishing crossover and bifurcation relationships between vessels

4. Filtering of the crossovers and bifurcations

\subsubsection{Tracking and labelling of vessel segments}

To detect and label the vessel segments, an image tracking process is performed. As the crease images eliminate background information, any non-null pixel (intensity greater than zero) belongs to a vessel segment. Taking this into account, each row in the image is tracked (from top to bottom) and when a non-null pixel is found, the segment tracking process takes place. The aim is to label the vessel segment found, as a line of 1 pixel width. This is, every pixel will have only two neighbors (previous and next) avoiding ambiguity to track the resulting segment in further processes.

To start the tracking process, the configuration of the 4 pixels which have not been analyzed by the initially detected pixel is calculated. This leads to 16 possible configurations depending on whether there is a segment pixel or not in each one of the 4 positions. If the initial pixel has no neighbors, it is discarded and the image tracking continues. In the other cases there are two main possibilities: either the initial pixel is an endpoint for the segment, so this is tracked in one way only or the initial pixel is a middle point and the segment is tracked in two ways from it. Figure 9 shows the 16 possible neighborhood configurations and how the tracking directions are established in any case.

Once the segment tracking process has started, in every step a neighbor of the last pixel flagged as segment is selected to be the next. This choice is made using the following criterion: the best neighbor is the one with the most non-flagged neighbors corresponding to segment pixels. This heuristic contains the idea of keeping the 1-pixel width segment to track along the middle of the crease, where pixels have more segment pixel neighbors. In case of a tie, the heuristic tries to preserve the most repeated orientation in the last steps. When the whole image tracking process finishes, every segment is a 1 pixel width line with its endpoints defined. The endpoints are very useful to establish relationships between segments because these relationships can always be detected in the surroundings of a segment endpoint. This avoids the analysis of every pixel belonging to a vessel, considerably reducing the complexity of the algorithm and therefore the running time.

\subsubsection{Union relationships}

As stated before, the union detection is needed to build the vessels out of their segments. Aside the segments from the crease image, no additional information is required and therefore is the first kind of relationship to be detected in the image. An union or joint between two segments exists when one of the segments is the continuation of the other in the same retinal vessel. Figure 10 shows some examples of union relationships between segments.

To find these relationships, the developed algorithm uses the segment endpoints calculated and labelled in the previous subsection. The main idea is to analyze pairs of close endpoints from different segments and quantify the likelihood of one being the prolongation of the other. The proposed algorithm connects both endpoints and measures the smoothness of the connection.

An efficient approach to connect the segments is using an straight line between both endpoints. In Figure 11, a graphical description of the detection process for an union is 


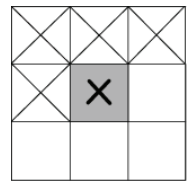

(a)

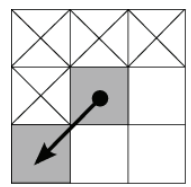

(e)

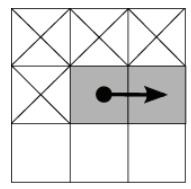

(i)

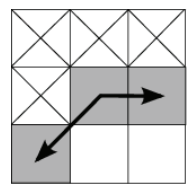

(m)

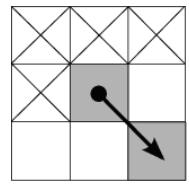

(b)

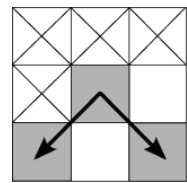

(f)

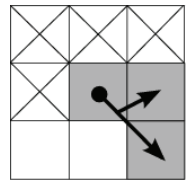

(j)

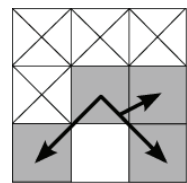

(n)

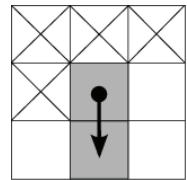

(c)

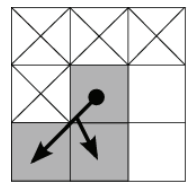

(g)

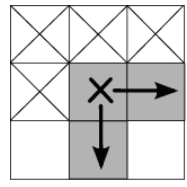

(k)

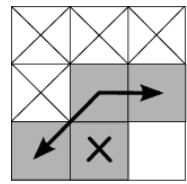

(o)

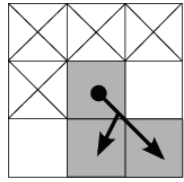

(d)

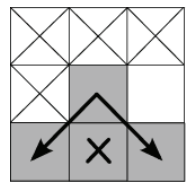

(h)

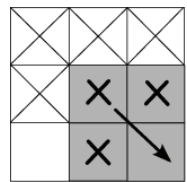

(1)

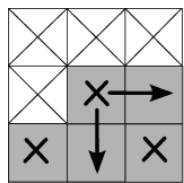

(p)

Fig. 9. Initial tracking process for a segment depending on the neighbor pixels surrounding the first pixel found for the new segment in a 8-neighborhood. As there are 4 neighbors not tracked yet (the bottom row and the one to the right), there are a total of 16 possible configurations. Gray squares represent crease (vessel) pixels and the white ones, background pixels. The upper row neighbors and the left one are ignored as they have already been tracked due to the image tracking direction. Arrows point to the next pixels to track while crosses flag pixels to be ignored. In 9(d), 9(g), 9(j) and 9(n) the forked arrows mean that only the best of the pointed pixels (i.e. the one with more new vessel pixel neighbors) is selected for continuing the tracking. Arrows starting with a black circle flag the central pixel as an endpoint for the segment (9(b), 9(c), 9(d), 9(e), 9(g), 9(i), 9(j)).

shown. The smoothness measurement is obtained from the angles between the straight line and the segment direction. The segment direction is calculated by the endpoint direction. The maximum smoothness occurs when both angles are $\pi$ rad., i.e. both segments are parallel and belong to the straight line connecting it. The smoothness decreases as both angles decrease. A criterion to accept the candidate relationship must be established. A minimum angle $\theta_{\min }$ is set as the threshold for both angles. This way, the criterion to accept an union relationship is defined as

$$
\operatorname{Union}(r, s)=\left(\alpha>\theta_{\text {min }}\right) \wedge\left(\beta>\theta_{\text {min }}\right)
$$




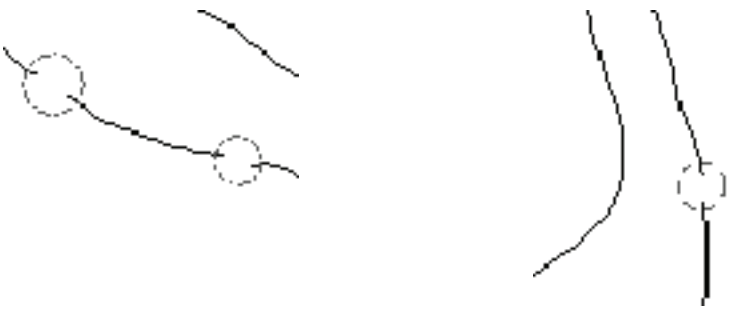

Fig. 10. Examples of union relationships. Some of the vessels present discontinuities leading to different segments. These discontinuities are detected in the union relationships detection process.

where $r, s$ are the segments involved in the union and $\alpha, \beta$ their respective endpoint directions. It has been observed that for values of $\theta_{\min }$ close to $\frac{3}{4} \pi$ rad. the algorithm delivers good results in all cases.

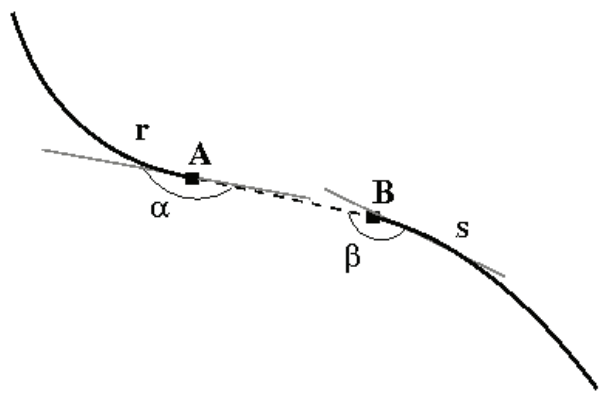

Fig. 11. Union of the crease segments $r$ and $s$. The angles between the new segment $\overline{A B}$ and the crease segments $r(\alpha)$ and $s(\beta)$ are near $\pi \mathrm{rad}$, so they are above the required threshold $\left(\frac{3}{4} \pi\right)$ and the union is finally accepted.

\subsubsection{Bifurcation/crossover relationships}

Bifurcations and crossovers are the feature interest points in this work for characterizing individuals by a biometric pattern. A crossover is an intersection between two segments. A bifurcation is a point in a segment where another one starts from. While unions allow to build the vessels, bifurcations allow to build the vessel tree by establishing relationships between them. Using both types, the retinal vessel tree can be reconstructed by joining all segments. An example of this is shown in Figure 12.

A crossover can be seen in the segment image, as two close bifurcations forking from the same segment. Therefore, finding bifurcation and crossover relationships between segments can be initially reduced to find only bifurcations. Crossovers can then be detected analyzing close bifurcations.

In order to find bifurcations in the image, an idea similar to the union algorithm is followed based on the search of the bifurcations from the segments endpoints. The criterion in this case is finding a segment close to an endpoint whose segment can be assumed to start in the found one. This way, the algorithm does not require to track the whole segments, bounding complexity to the number of segments and not to their length. 


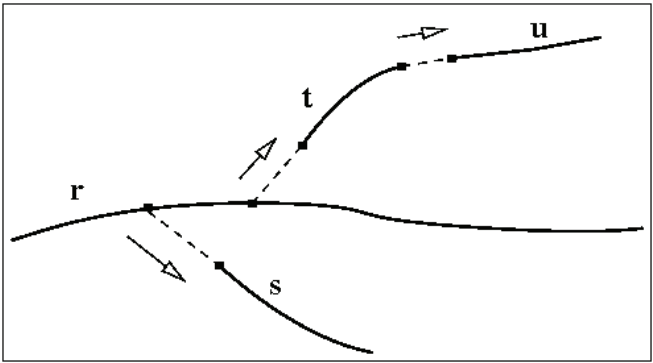

Fig. 12. Retinal Vessel Tree reconstruction by unions $(t, u)$ and bifurcations $(r, s)$ and $(r, t)$.

For every endpoint in the image, the process is as follows (Figure 13):

1. Compute the endpoint direction.

2. Extend the segment in that direction a fixed length $l_{\max }$.

3. Analyze the points in and nearby the prolongation segment to find candidate segments.

4. If a point of a different segment is found, compute the angle $(\alpha)$ associated to that bifurcation, defined by the direction of this point and the extreme direction from step 1.

The parameter $l_{\max }$ is inserted in the model to avoid indefinite prolongation of the segments. If it follows that $l<=l_{\max }$, the segments will be joined and a bifurcation will be detected, being $l$ the distance from the endpoint of the segment to the other segment.

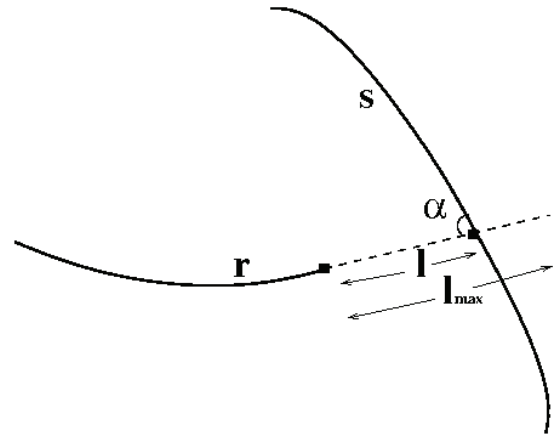

Fig. 13. Bifurcation between segment $r$ and $s$. The endpoint of $r$ is prolonged a maximum distance $l_{\max }$ and eventually a point of segment $s$ is found.

Figure 14 shows an example of results after this stage where feature points are marked. Also, spurious detected points are identified in the image. These spurious points may occur for different reasons such as wrongly detected segments. In the image test set used (over 100 images) the approximate mean number of feature points detected per image was 28 . The mean of spurious points corresponded to 5 points per image. To improve the performance of the matching process is convenient to eliminate as spurious points as possible. Thus, the last stage in the biometric pattern extraction process will be the filtering of spurious points in order to obtain an accurate biometric pattern for an individual. 


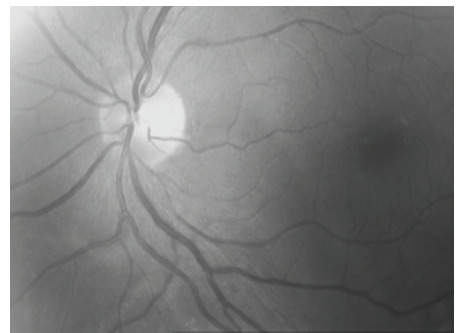

(a)

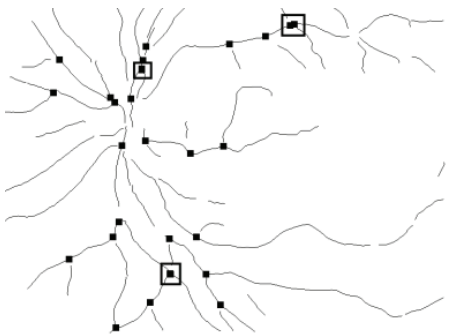

(b)

Fig. 14. Example of feature points extracted from original image after the bifurcation/crossover stage. (a) Original Image. (b) Feature points marked over the segment image. Spurious points corresponding to the same crossover (detected as two bifurcations) are signalled in squares.

\subsubsection{Filtering of feature points}

A segment filtering process takes place in the tracking stage, filtering detected segments by their length using a threshold, $T_{\min }$. This leads to images with minimum false segments and with only important segments in the vessel tree.

Finally, since crossover points are detected as two bifurcation points, as Figure 14(b) shows, these bifurcation points are merged into an unique feature point by calculating the midpoint between them.

Figure 15 shows an example of the filtering process result, i.e. the biometric pattern obtained from an individual. Briefly, in the initial test set of images used to tune the parameters, the reduction of false detected points was about from 5 to 2 in the average.

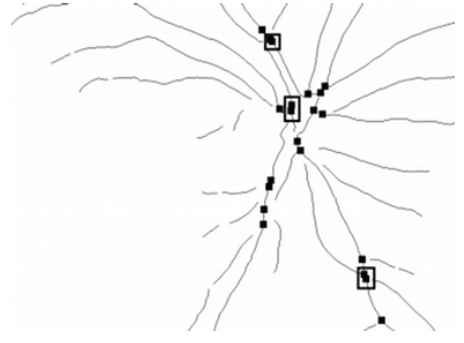

(a)

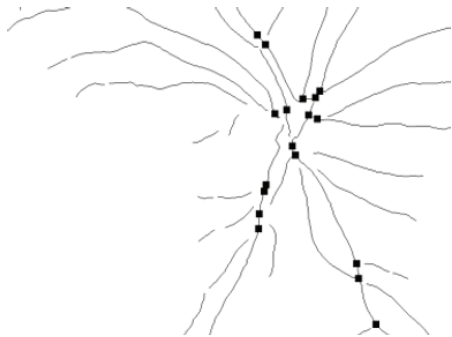

(b)

Fig. 15. Example of the result after the feature point filtering. (a) Image containing feature points before filtering. (b) Image containing feature points after filtering. Spurious points from duplicate crossover points have been eliminated.

\subsection{Biometric pattern matching}

In the matching stage, the stored reference pattern, $v$, for the claimed identity is compared to the pattern extracted, $v^{\prime}$, during the previous stage. Due to the eye movement during the image acquisition stage, it is necessary to align $v^{\prime}$ with $v$ in order to be matched L.G.Brown (1992); M.S.Markov et al. (1993); Zitová \& Flusser (2003). This fact is illustrated in Figure 16 
where two images from the same individual, 16(a) and 16(c), and the obtained results in each case, 16(b) and 16(d), are shown using the crease approach.

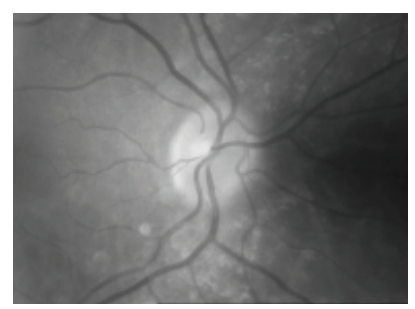

(a)

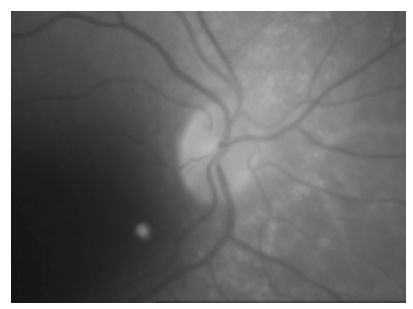

(c)

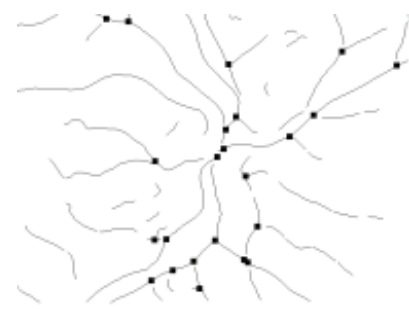

(b)

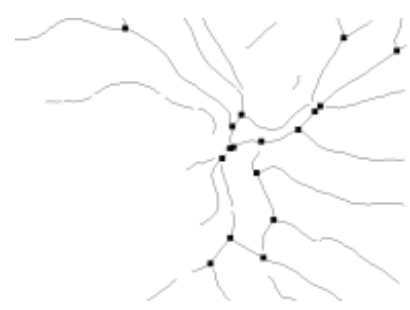

(d)

Fig. 16. Examples of feature points obtained from images of the same individual acquired in different times. (a) and (c) original images. (b) Feature point image from (a). A set of 23 points is obtained. (d) Feature point image from (c). A set of 17 points are obtained.

Depending on several factors, such as the eye location in the objective, patterns may suffer some deformations. A reliable and efficient model is necessary to deal with these deformations allowing to transform the candidate pattern in order to get a pattern similar to the reference one. The movement of the eye in the image acquisition process basically consists in translation in both axis, rotation and sometimes a very small change in scale. It is also important to note that both patterns $v$ and $v^{\prime}$ could have a different number of points even being from the same individual. This is due to the different conditions of illumination and orientation in the image acquisition stage.

The transformation considered in this work is the Similarity Transformation (ST), which is a special case of the Global Affine Transformation (GAT). ST can model translation, rotation and isotropic scaling using 4 parameters Ryan et al. (2004). The ST works fine with this kind of images as the rotation angle is moderate. It has also been observed that the scaling, due to eye proximity to the camera, is nearly constant for all the images. Also, the rotations are very slight as the eye orientation when facing the camera is very similar. Under these circumstances, the ST model appears to be very suitable.

The ultimate goal is to achieve a final value indicating the similarity between the two feature points set, in order to decide about the acceptance or the rejection of the hypothesis that both images correspond to the same individual. To develop this task the matching pairings between both images must be determined. A transformation has to be applied to the candidate image in order to register its feature points with respect to the corresponding points in the reference image. The set of possible transformations is built based on some restrictions and 
a matching process is performed for each one of these. The transformation with the highest matching score will be accepted as the best transformation.

To obtain the four parameters of a concrete ST, two pairs of feature points between the reference and candidate patterns are considered. If $M$ is the total number of feature points in the reference pattern and $N$ the total number of points in the candidate one, the size of the set $T$ of possible transformations is computed using Eq.(2):

$$
T=\frac{\left(M^{2}-M\right)\left(N^{2}-N\right)}{2}
$$

where $M$ and $N$ represent the cardinality of $v$ and $v^{\prime}$ respectively.

Since $T$ represents a high number of transformations, some restrictions must be applied in order to reduce it. As the scale factor between patterns is always very small in this acquisition process, a constraint can be set to the pairs of points to be associated. In this scenario, the distance between both points in each pattern has to be very similar. As it cannot be assumed that it will be the same, two thresholds are defined, $S_{\min }$ and $S_{\max }$, to bound the scale factor. This way, elements from $T$ are removed where the scale factor is greater or lower than the respective thresholds $S_{\min }$ and $S_{\max }$. Eq.(3) formalises this restriction:

$$
S_{\min }<\frac{\operatorname{distance}(p, q)}{\operatorname{distance}\left(p^{\prime}, q^{\prime}\right)}<S_{\max }
$$

where $p, q$ are points from $v$ pattern, and $p^{\prime}, q^{\prime}$ are the matched points from the $v$ pattern. Using this technique, the number of possible matches greatly decrease and, in consequence, the set of possible transformations decreases accordingly. The mean percentage of not considered transformations by these restrictions is around $70 \%$.

In order to check feature points, a similarity value between points (SIM) is defined which indicates how similar two points are. The distance between these two points will be used to compute that value. For two points $A$ and $B$, their similarity value is defined by Eq.(4):

$$
\operatorname{SIM}(A, B)=1-\frac{\operatorname{distance}(A, B)}{D_{\max }}
$$

where $D_{\max }$ is a threshold that stands for the maximum distance allowed for those points to be considered a possible match. If $\operatorname{distance}(A, B)>D_{\max }$ then $\operatorname{SIM}(A, B)=0 . D_{\max }$ is a threshold introduced in order to consider the quality loss and discontinuities during the creases extraction process leading to mislocation of feature points by some pixels.

In some cases, two points $B_{1}, B_{2}$ could have both a good value of similarity with one point $A$ in the reference pattern. This happens because $B_{1}$ and $B_{2}$ are close to each other in the candidate pattern. To identify the most suitable matching pair, the possibility of correspondence is defined comparing the similarity value between those points to the rest of similarity values of each one of them:

$$
P\left(A_{i}, B_{j}\right)==\frac{\operatorname{SIM}\left(A_{i}, B_{j}\right)^{2}}{\left(\sum_{i \prime=1}^{M} \operatorname{SIM}\left(A_{i^{\prime}}, B_{j}\right)+\sum_{j^{\prime}=1}^{N} \operatorname{SIM}\left(A_{i}, B_{j^{\prime}}\right)-\operatorname{SIM}\left(A_{i}, B_{j}\right)\right)}
$$


A $M \times N$ matrix $Q$ is constructed such that position $(i, j)$ holds $P\left(A_{i}, B_{j}\right)$. Note that if the similarity value is 0 , the possibility value is also 0 . This means that only valid matchings will have a non-zero value in $Q$. The desired set $C$ of matching feature points is obtained from $P$ using a greedy algorithm. The element $(i, j)$ inserted in $C$ is the position in $Q$ where the maximum value is stored. Then, to prevent the selection of the same point in one of the images again, the row $(i)$ and the column $(j)$ associated to that pair are set to 0 . The algorithm finishes when no more non-zero elements can be selected from $Q$.

The final set of matched points between patterns is C. Using this information, a similarity metric must be established to obtain a final criterion of comparison between patterns.

\section{Similarity metrics analysis}

The goal in this stage of the process is to define similarity measures on the aligned patterns to correctly classify authentications in both classes: attacks (unauthorised accesses), when the two matched patterns are from different individuals and clients (authorised accesses) when both patterns belong to the same person.

For the metric analysis a set of 150 images (100 images, 2 images per individual and 50 different images more) from VARIA database VARIA (2007) were used. The rest of the images will be used for testing in the next section. The images from the database have been acquired with a TopCon non-mydriatic camera NW-100 model and are optic disc centred with a resolution of $768 \times 584$. There are 60 individuals with two or more images acquired in a time span of 6 years. These images have a high variability in contrast and illumination allowing the system to be tested in quite hard conditions. In order to build the training set of matchings, all images are matched versus all the images (a total of 150x150 matchings) for each metric. The matchings are classified into attacks or clients accesses depending if the images belong to the same individual or not. Distributions of similarity values for both classes are compared in order to analyse the classification capabilities of the metrics.

The main information to measure similarity between two patterns is the number of feature points successfully matched between them. Fig.17(a) shows the histogram of matched points for both classes of authentications in the training set. As it can be observed, matched points information is by itself quite significative but insufficient to completely separate both populations as in the interval $[10,13]$ there is an overlapping between them.

This overlapping is caused by the variability of the patterns size in the training set because of the different illumination and contrast conditions in the acquisition stage. Fig.17(b) shows the histogram for the biometric pattern size, i.e. the number of feature points detected. A high variability can be observed, as some patterns have more than twice the number of feature points of other patterns. As a result of this, some patterns have a small size, capping the possible number of matched points (Fig. 18). Also, using the matched points information alone lacks a well bounded and normalised metric space.

To combine information of patterns size and normalise the metric, a function $f$ will be used. Normalised metrics are very common as they make easier to compare class separability or establishing valid thresholds. The similarity measure $(S)$ between two patterns will be defined by

$$
S=\frac{C}{f(M, N)}
$$




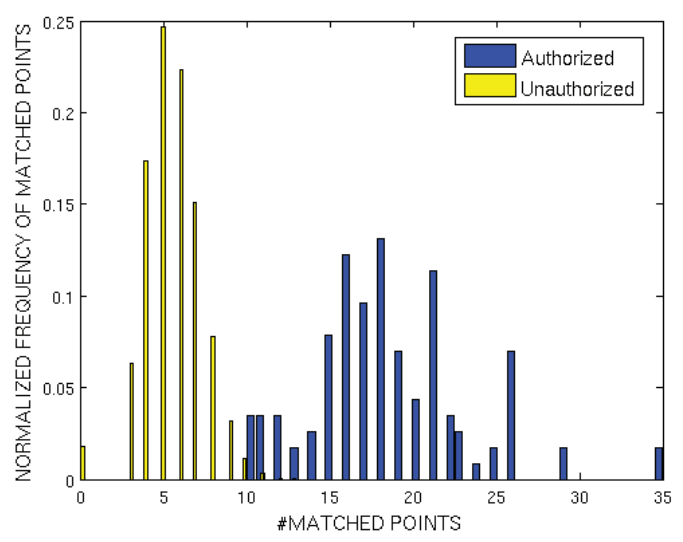

(a)

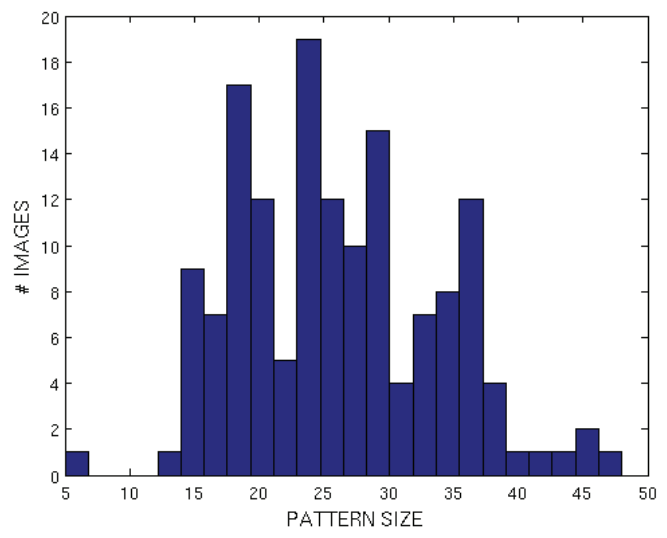

(b)

Fig. 17. (a) Matched points histogram in the attacks (unauthorised) and clients (authorised) authentications cases. In the interval $[10,13]$ both distributions overlap. (b) histogram of detected points for the patterns extracted from the training set.

where $C$ is the number of matched points between patterns, and $M$ and $N$ are the matching patterns sizes. The first $f$ function defined and tested is:

$$
f(M, N)=\min (M, N)
$$

The min function is the less conservative as it allows to obtain a maximum similarity even in cases of different sized patterns. Fig.19(a) shows the distributions of similarity scores for clients and attacks classes in the training set using the normalisation function defined in Eq.(7), and Fig.19(b) shows the FAR and FRR curves versus the decision threshold. 


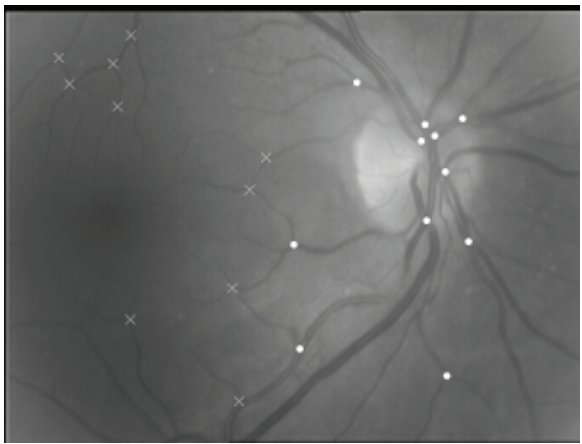

(a)

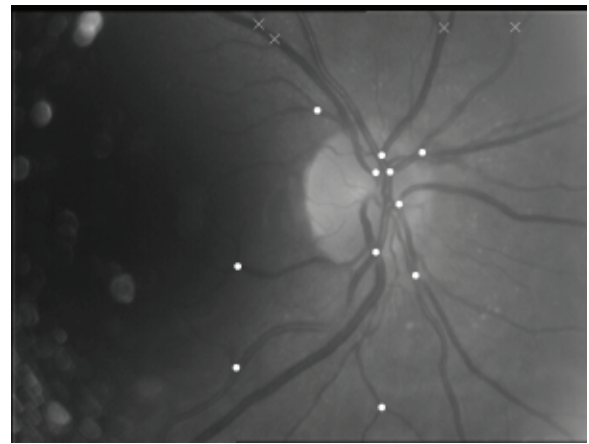

(b)

Fig. 18. Example of matching between two samples from the same individual in VARIA database. White circles mark the matched points between both images while crosses mark the unmatched points. In (b) the illumination conditions of the image lead to miss some features from left region of the image. Therefore, a small amount of detected feature points is obtained capping the total amount of matched points.

Although the results are good when using the normalisation function defined in Eq.(7), a few cases of attacks show high similarity values, overlapping with the clients class. This is caused by matchings involving patterns with a low number of feature points as $\min (M, N)$ will be very small, needing only a few points to match in order to get a high similarity value. This suggests, as it will be reviewed in section 5 , that some minimum quality constraint in terms of detected points would improve performance for this metric.

To improve the class separability, a new normalisation function $f$ is defined:

$$
f(M, N)=\sqrt{M N}
$$

Fig.20(a) shows the distributions of similarity scores for clients and attacks classes in the training set using the normalisation function defined in Eq.(8) and Fig.20(b) shows the FAR and FRR curves versus the decision threshold.

Function defined in Eq.(8) combines both patterns size in a more conservative way, preventing the system to obtain a high similarity value if one pattern in the matching process contains a low number of points. This allows to reduce the attacks class variability and, moreover, to separate its values away from the clients class as this class remains in a similar values range. As a result of the new attacks class boundaries, a decision threshold can be safely established where $F A R=F R R=0$ in the interval $[0.38,0.5]$ as Fig.20(b) clearly exposes. Although this metric shows good results, it also has some issues due to the normalisation process which can be corrected to improve the results as showed in next subsection.

\subsection{Confidence band improvement}

Normalising the metric has the side effect of reducing the similarity between patterns of the same individual where one of them had a much greater number of points than the other, even in cases with a high number of matched points. This means that some cases easily distinguishable based on the number of matched points are now near the confidence band borders. To take a closer look at this region surrounding the confidence band, the cases of 


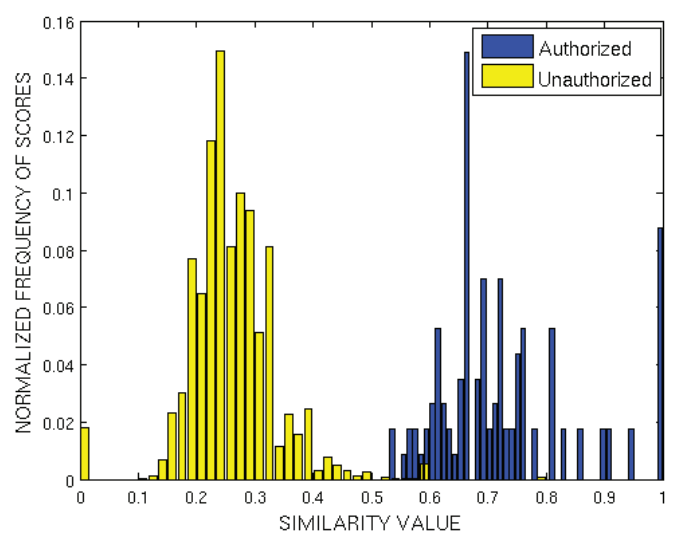

(a)

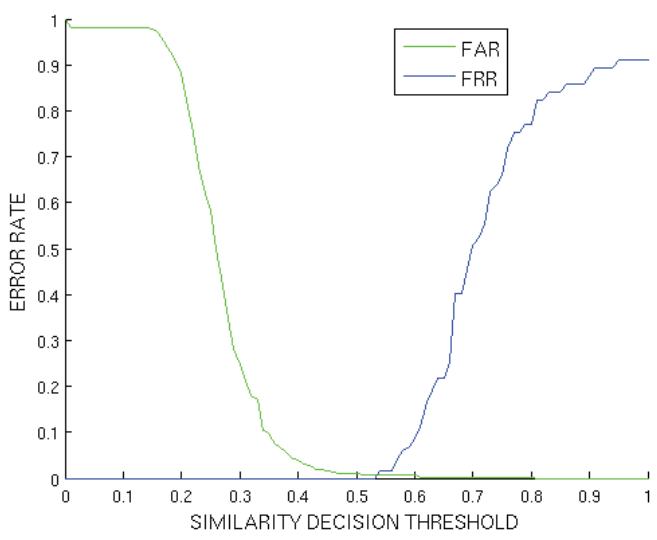

(b)

Fig. 19. (a) Similarity values distribution for authorised and unauthorised accesses using $f=\min (M, N)$ as normalisation function for the metric. (b) False Accept Rate (FAR) and False Rejection Rate (FRR) for the same metric.

unauthorised accesses with the highest similarity values $(S)$ and authorised accesses with the lowest ones are evaluated. Fig.21 shows the histogram of matched points for cases in the marked region of Fig.20(b). It can be observed that there is an overlapping but both histograms are highly distinguishable.

To correct this situation, the influence of the number of matched points and the patterns size have to be balanced. A correction parameter $(\gamma)$ is introduced in the similarity measure to control this. The new metric is defined as:

$$
S_{\gamma}=S \cdot C^{\gamma-1}=\frac{C^{\gamma}}{\sqrt{M N}}
$$




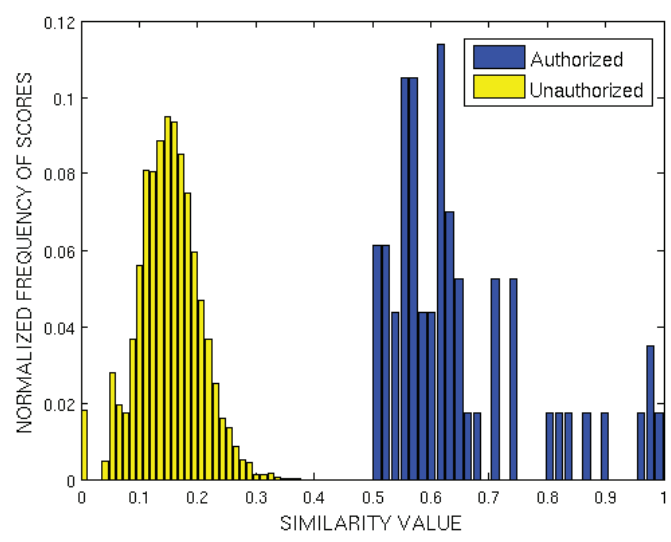

(a)

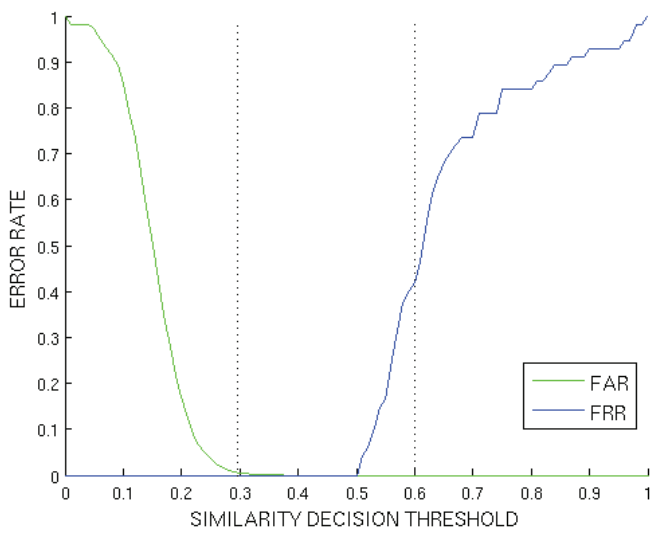

(b)

Fig. 20. (a) similarity values distribution for authorised and unauthorised accesses using $f=\sqrt{M N}$ as normalisation function for the metric. (b) False Accept Rate (FAR) and False Rejection Rate (FRR) for the same metric. Dotted lines delimit the interest zone surrounding the confidence band which will be used for further analysis.

with $S, C, M$ and $N$ the same parameters from Eq.(8). The $\gamma$ correction parameter allows to improve the similarity values when a high number of matched points is obtained, specially in cases of patterns with a high number of points.

Using the gamma parameter, values can be higher than 1 . In order to normalise the metric back into a $[0,1]$ values space, a sigmoid transference function, $T(x)$, is used:

$$
T(x)=\frac{1}{1+e^{s \cdot(x-0.5)}}
$$




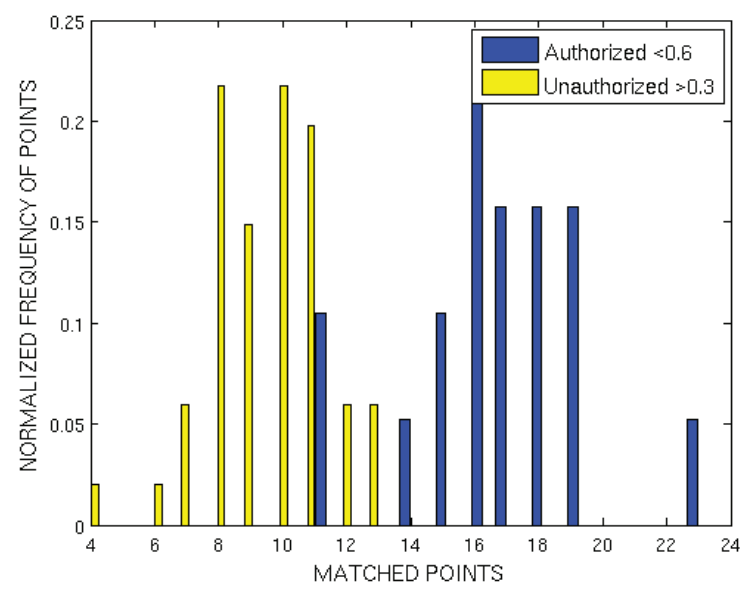

Fig. 21. Histogram of matched points in the populations of attacks whose similarity is higher than 0.3 and clients accesses whose similarity is lower than 0.6.

where $\mathrm{s}$ is a scale factor to adjust the function to the correct domain as $S_{\gamma}$ does not return negatives or much higher than 1 values when a typical $\gamma \in[1,2]$ is used. In this work, $s=6$ was chosen empirically. The normalised gamma-corrected metric, $S_{\gamma}^{\prime}(x)$, is defined by:

$$
S_{\gamma}^{\prime}=T\left(S_{\gamma}\right)
$$

Finally, to choose a good $\gamma$ parameter, the confidence band improvement has been evaluated for different values of $\gamma$ (Fig.22(a)). The maximum improvement is achieved at $\gamma=1.12$ with a confidence band of 0.3288 , much higher than the original from previous section. The distribution of the whole training set (using $\gamma=1.12$ ) is showed in Fig.22(b) where the wide separation between classes can be observed.

\section{Results}

A set of 90 images, 83 different from the training set and 7 from the previous set with the highest number of points, has been built in order to test the metrics performance once their parameters have been fixed with the training set. To test the metrics performance, the False Acceptance Rate and False Rejection Rate were calculated for each of them (the metrics normalised by Eq.(7), Eq.(8) and the gamma-corrected normalised metric defined in Eq.(11). A usual error measure is the Equal Error Rate (EER) that indicates the error rate where FAR curve and FRR curve intersect. Fig.23(a) shows the FAR and FRR curves for the three previously specified metrics. The EER is 0 for the normalised by geometrical mean (MEAN) and gamma corrected (GAMMA) metrics as it was the same case in the training set, and, again, the gamma corrected metric shows the highest confidence band in the test set, 0.2337.

The establishment of a wide confidence band is specially important in this scenario of different images from users acquired on different times and with different configurations of the capture hardware.

Finally, to evaluate the influence of the image quality, in terms of feature points detected per image, a test is run where images with a biometric pattern size below a threshold are removed 


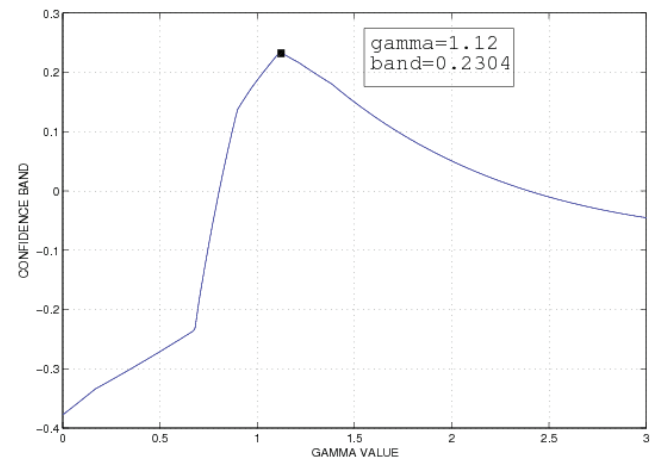

(a)

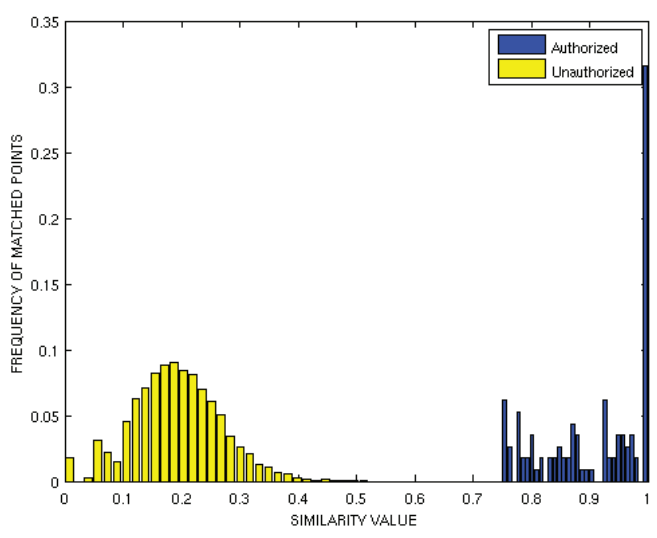

(b)

Fig. 22. (a) Confidence band size vs gamma $(\gamma)$ parameter value. Maximum band is obtained at $\gamma=1.12$. (b) Similarity values distributions using the normalised metric with $\gamma=1.12$.

for the set and the confidence band obtained with the rest of the images is evaluated. Fig.23(b) shows the evolution of the confidence band versus the minimum detected points constraint. The confidence band does not grow significatively until a fairly high threshold is set. Taking as threshold the mean value of detected points for all the test set, 25.2, the confidence band grows from 0.2337 to 0.3317 . So removing half of the images, the band is increased only by 0.098 suggesting that the gamma-corrected metric is very robust to low quality images.

The mean execution time on a 2.4Ghz. Intel Core Duo desktop PC for the authentication process, implemented in $\mathrm{C}++$, was $155 \mathrm{~ms}$ : $105 \mathrm{~ms}$ in the feature extraction stage and $50 \mathrm{~ms}$ in the registration and similarity measure estimation, so that the method is very well-fitted to be employed in a real verification system. 


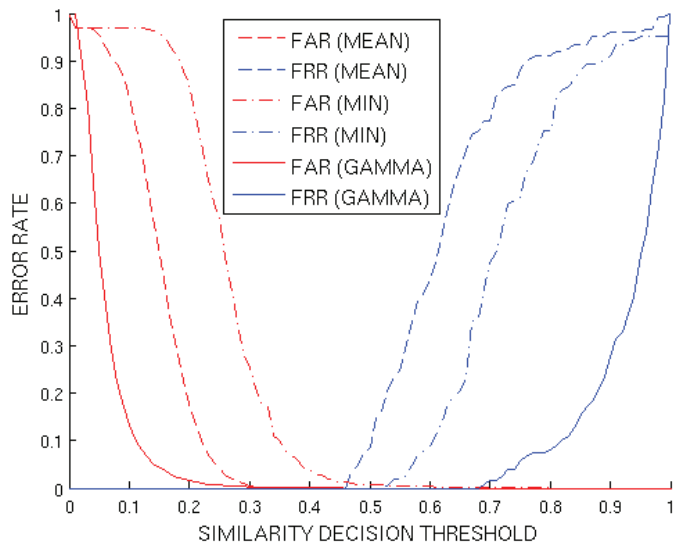

(a)

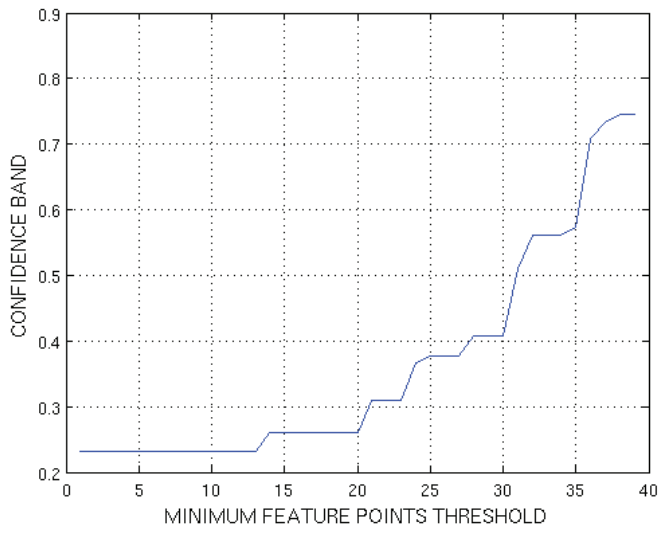

(b)

Fig. 23. (a) FAR and FRR curves for the normalised similarity metrics (min: normalised by minimum points, mean: normalised by geometrical mean and gamma: gamma corrected metric). The best confidence band is the one belonging to the gamma corrected metric corresponding to 0.2337.(b) Evolution of the confidence band using a threshold of minimum detected points per pattern. 


\section{Conclusions and future work}

In this work a complete identity verification method has been introduced. Following the same idea as the fingerprint minutiae-based methods, a set of feature points is extracted from digital retinal images. This unique pattern will allow for the reliable authentication of authorised users. To get the set of feature points, a creases-based extraction algorithm is used. After that, a recursive algorithm gets the point features by tracking the creases from the localised optic disc. Finally, a registration process is necessary in order to match the reference pattern from the database and the acquired one. With the patterns aligned, it is possible to measure the degree of similarity by means of a similarity metric. Normalised metrics have been defined and analysed in order to test the classification capabilities of the system. The results are very good and prove that the defined authentication process is suitable and reliable for the task. The use of feature points to characterise individuals is a robust biometric pattern allowing to define metrics that offer a good confidence band even in unconstrained environments when the image quality variance can be very high in terms of distortion, illumination or definition. This is also possible as this methodology does not rely on the localisation or segmentation of some reference structures, as it might be the optic disc. Thus, if the the user suffers some structure distorting pathology and this structure cannot be detected, the system works the same with the only problem being a possible loss of feature points constrained to that region. Future work includes the use of some high-level information of points to complement metrics performance and new ways of codification of the biometric pattern allowing to perform faster matches.

\section{References}

Bolle, R. M., Senior, A. W., Ratha, N. K. \& Pankanti, S. (2002). Fingerprint minutiae: A constructive definition, Biometric Authentication, pp. 58-66.

Caderno, I. G., Penedo, M. G., Mariño, C., Carreira, M. J., Gómez-Ulla, F. \& González, F. (2004). Automatic extraction of the retina av index, ICIAR (2), pp. 132-140.

Chou, C.-T., Shih, S.-W. \& Chen, D.-Y. (2006). Design of gabor filter banks for iris recognition, IIH-MSP, pp. 403-406.

C.Mariño, M.G.Penedo, M.J.Carreira \& F.Gonzalez (2003). Retinal angiography based authentication, Lecture Notes in Computer Science 2905: 306-313.

De Schaepdrijver, L., Simoens, L., Lauwers, H. \& DeGesst, J. (1989). Retinal vascular patterns in domestic animals, Res. Vet. Sci. 47: 34-42.

Farzin, H., Abrishami-Moghaddam, H. \& Moin, M.-S. (2008). A novel retinal identification system, EURASIP Journal on Advances in Signal Processing ID 280635: 10 pp.

He, Z., Sun, Z., Tan, T., Qiu, X., Zhong, C. \& Dong, W. (2008). Boosting ordinal features for accurate and fast iris recognition, $C V P R$.

Hill, R. (1999). Retina identification, in A. Jain, R. Bolle \& S. Pankanti (eds), Biometrics: Personal Identification in Networked Society, Kluwer Academic Press, Boston, pp. 123-142.

Jain, A. K., Ross, A. \& Pankanti, S. (1999). A prototype hand geometry-based verification system, AVBPA, pp. 166-171.

Kim, H.-C., Kim, D., Bang, S. Y. \& Lee, S.-Y. (2004). Face recognition using the second-order mixture-of-eigenfaces method, Pattern Recognition 37(2): 337-349.

Kim, J., Cho, S., Choi, J. \& Marks, R. J. (2004). Iris recognition using wavelet features, VLSI Signal Processing 38(2): 147-156. 
Kisku, D. R., Rattani, A., Tistarelli, M. \& Gupta, P. (2008). Graph application on face for personal authentication and recognition, ICARCV, pp. 1150-1155.

L.G.Brown (1992). A survey of image registration techniques, ACM Computer Surveys 24(4): 325-376.

Lloret, D., López, A., Serrat, J. \& Villanueva, J. (1999). Creaseness-based CT and MR registration: comparison with the mutual information method, Journal of Electronic Imaging 8(3): 255-262.

Lloret, D., Mariño, C., Serrat, J., A.M.López \& Villanueva, J. (2001). Landmark-based registration of full SLO video sequences, Proceedings of the IX Spanish Symposium on Pattern Recognition and Image Analysis, Vol. I, pp. 189-194.

López, A., Lloret, D., Serrat, J. \& Villanueva, J. (2000). Multilocal creaseness based on the level-set extrinsic curvature, Computer Vision and Image Understanding 77(1): 111-144.

López, A., Lumbreras, F., Serrat, J. \& Villanueva, J. (1999). Evaluation of methods for ridge and valley detection, IEEE Trans. on Pattern Analysis and Machine Intelligence 21(4): 327-335.

López, A. M., Lumbreras, F. \& Serrat, J. (1998). Creaseness from level set extrinsic curvature, ECCV, pp. 156-169.

Ma, L., Wang, Y. \& Tan, T. (2002). Iris recognition using circular symmetric filters, ICPR (2), pp. 414-417.

Maio, D. \& Maltoni, D. (1997). Direct gray-scale minutiae detection in fingerprints, IEEE Trans. Pattern Anal. Mach. Intell. 19(1): 27-40.

Mariño, C., Penedo, M. G., Penas, M., Carreira, M. J. \& Gonzalez, F. (2006). Personal authentication using digital retinal images, Pattern Analysis and Applications 9:21-33.

Mian, A. S., Bennamoun, M. \& Owens, R. A. (2008). Keypoint detection and local feature matching for textured $3 \mathrm{~d}$ face recognition, International Journal of Computer Vision 79(1): 1-12.

Moghaddam, B. \& Pentland, A. (1997). Probabilistic visual learning for object representation, IEEE Trans. Pattern Anal. Mach. Intell. 19(7): 696-710.

M.S.Markov, H.G.Rylander \& A.J.Welch (1993). Real-time algorithm for retinal tracking, IEEE Trans. on Biomedical Engineering 40(12): 1269-1281.

Nabti, M. \& Bouridane, A. (2007). An improved iris recognition system using feature extraction based on wavelet maxima moment invariants, ICB, pp. 988-996.

Noden, D. (1989). Embryonic origins and assembly of blood vessels, Am. Rev. Respir. Dis. 140: 1097-1103.

Ryan, N., Heneghan, C. \& de Chazal, P. (2004). Registration of digital retinal images using landmark correspondence by expectation maximization, Image and Vision Computing 22: 883-898.

Seung-Hyun, L., Sang-Yi, Y. \& Eun-Soo, K. (1995). Fingerprint identification by use of a volume holographic optical correlator, Proc. SPIE Vol. 3715, Optical Pattern Recognition pp. 321-325.

Sidlauskas, D. (1988). 3d hand profile identification apparatus, United States Patent No.4,736.203.

Siguenza Pizarro, J. A. \& Tapiador Mateos, M. (2005). Tecnologias Biometricas Aplicadas a Seguridad, Ra-Ma.

Simon, C. \& Goldstein, I. (1935). A new scientific method of identification, J. Medicine 35(18): 901-906.

systems Lab, B. (n.d.). Hasis, a hand shape identification system. 
Tower, P. (1955). The fundus oculi in monozygotic twins: Report of six pairs of identical twins, Arch. Ophthalmol. 54: 225-239.

VARIA (2007). VARPA Retinal Images for Authentication. http://www.varpa.es/varia.html.

Venkataramani, K. \& Kumar, V. (2003). Fingerprint verification using correlation filters, AVBPA, pp. 886-894.

Whittier, J. C., Doubet, J., Henrickson, D., Cobb, J., Shadduck, J. \& Golden, B. (2003). Biological considerations pertaining to use of the retinal vascular pattern for permanent identification of livestock, J. Anim. Sci 81: 1-79.

Yang, M.-H., Ahuja, N. \& Kriegman, D. J. (2000). Face recognition using kernel eigenfaces, ICIP.

Zitová, B. \& Flusser, J. (2003). Image registration methods: a survey, Image Vision and Computing 21(11): 977-1000.

Zunkel, R. (1999). Hand geometry based verification, BIOMETRICS:Personal Identification in Networked Society, Kluwert Academic Publishers. 


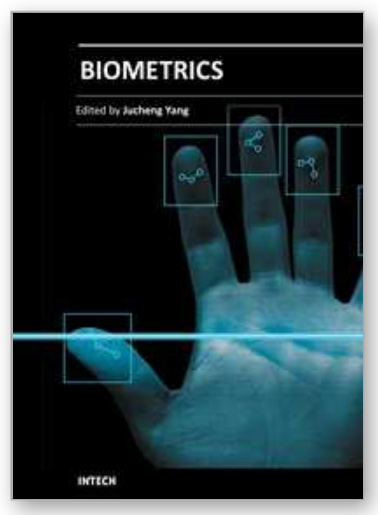

\author{
Biometrics \\ Edited by Dr. Jucheng Yang
}

ISBN 978-953-307-618-8

Hard cover, 266 pages

Publisher InTech

Published online 20, June, 2011

Published in print edition June, 2011

Biometrics uses methods for unique recognition of humans based upon one or more intrinsic physical or behavioral traits. In computer science, particularly, biometrics is used as a form of identity access management and access control. It is also used to identify individuals in groups that are under surveillance. The book consists of 13 chapters, each focusing on a certain aspect of the problem. The book chapters are divided into three sections: physical biometrics, behavioral biometrics and medical biometrics. The key objective of the book is to provide comprehensive reference and text on human authentication and people identity verification from both physiological, behavioural and other points of view. It aims to publish new insights into current innovations in computer systems and technology for biometrics development and its applications. The book was reviewed by the editor Dr. Jucheng Yang, and many of the guest editors, such as Dr. Girija Chetty, Dr. Norman Poh, Dr. Loris Nanni, Dr. Jianjiang Feng, Dr. Dongsun Park, Dr. Sook Yoon and so on, who also made a significant contribution to the book.

\title{
How to reference
}

In order to correctly reference this scholarly work, feel free to copy and paste the following:

Marcos Ortega and Manuel González Penedo (2011). Retinal vessel tree as biometric pattern, Biometrics, Dr. Jucheng Yang (Ed.), ISBN: 978-953-307-618-8, InTech, Available from:

http://www.intechopen.com/books/biometrics/retinal-vessel-tree-as-biometric-pattern

\section{INTECH}

open science | open minds

\section{InTech Europe}

University Campus STeP Ri

Slavka Krautzeka 83/A

51000 Rijeka, Croatia

Phone: +385 (51) 770447

Fax: +385 (51) 686166

www.intechopen.com

\section{InTech China}

Unit 405, Office Block, Hotel Equatorial Shanghai No.65, Yan An Road (West), Shanghai, 200040, China 中国上海市延安西路65号上海国际贵都大饭店办公楼405单元 Phone: +86-21-62489820

Fax: $+86-21-62489821$ 
(C) 2011 The Author(s). Licensee IntechOpen. This chapter is distributed under the terms of the Creative Commons Attribution-NonCommercialShareAlike-3.0 License, which permits use, distribution and reproduction for non-commercial purposes, provided the original is properly cited and derivative works building on this content are distributed under the same license. 